and 7 (1-10). Neonatal seizures were the most common presenting symptom, occurring in $7(60 \%)$ infants. Most infants were symptomatic by the second day. Eleven survived the neonatal period. None developed hydrocephalus. The diagnosis of ICH was confirmed by CAT scan. In most cases the hemorrhage was in the subarachnoid space and the CSF obtained by lumbar puncture contained blood. Obstetrical risk factors were: 1) prolonged second stage of labor ( $>2$ hrs), 2 ) very rapid labor with rapid descent, and 3) Pitocin and forceps delivery. CAT scan or ultrasound were considered essential for diagnosis of ICH. ( Sachs BP, Acker D et al. The incidence of symptomatic intracranial hemorrhage in term appropriate-for-gestation-age infants. Clinical Pediatrics 1987; 26:355).

COMMENT: Primary subarachnoid hemorrhage (SAH) was the most cormon form of hemorrhage in a previous study of 22 term newborn infants seen in a 5 year period with intracranial hemorrhage on CT examination (Fenichel GM et al. Arch Neurol 1984;4l:30). All infants with diffuse SAH had seizures on the first day of life. Five of 10 infants with SAH were born after prolonged labors, traumatic deliveries, and intrauterine asphyxia; three had intrauterine asphyxia. Five of 8 infants with intraventicular hemorrhage (IVH) had difficult deliveries with trauma and asphyxia. In 3 infants with IVH and 3 with intracerebral hemorrhage (ICH) the cause was unexplained, i.e. in almost one-third of the total group. The occurrence of intracranial hemorrhage does not preclude a good outcome. Development was normal or near normal at follow-up in one-half the infants who suffered a SAH and in 3 of 7 with IVH. Seizures in the first week of life are an important sign of SAH, IVH and ICH, occurring in almost all cases.

\title{
PROGNOSIS OF NEONATAL ASPHYXIA
}

Of 86 asphyxiated full-term neonates with CNS complications admitted to the Intensive Care Unit at the Nagoya City University Hospital, Japan, during a 10 year period (1972-81), 63 (738) survived the neonatal period. The CNS complications were hypoxic-ischemic encephalalopathy (58), intracranial hemorrhage (27) and brain infarction (1). Of 54 survivors who were followed for 3 to 13 years, 8 ( $15 \%$ ) had major handicaps such as cerebral palsy, epilepsy and mental retardation and 5 ( $9 \%$ ) had minor CNS abnormalities. Factors predictive of long-term CNS sequelae were: 1) absent Moro-reflex over 6-days, and 2) abnormal neurological signs on discharge.

Each neonate had a 1 min Apgar score of 6 or less, neurological abnormalities including stupor or lethargy, hypotonia, abnormal respirations, jitteriness or seizures, and one or more abnormal findings on laboratory tests such as spinal tap, echoencephalograpy, cerebral angiography, EEG, and CAT scan. ( Ogawa IT, Kanayama M, Wada Y. Long-term prognosis of asphyxiated full-term neonates with CNS complications. Brain Dev $1987 ; \underline{9}: 48-53$ ).

COMMENT: Another method of assessment of neurologic outcome in asphyxiated term infants is by use of serial CK-BB, or creatine kinase brain-type isoenzyme measurement (Walsh P. J Pediat 1982; 101:988). Serum CK-BB activity, when measured in cord blood and at 6-12 hours of life, correlates with neurologic outcome after severe asphyxia, comparing favorably with CT scanning as a prognostic factor. The mean of elevations in $\mathrm{CK}-\mathrm{BB}$ in asphyxiated was 4 -fold the values obtained in control infants. Normal CK-BB activity was a predictor of good neurologic outcome. 